\title{
Diagnostic value of BAL lymphocytosis in patients with indeterminate for usual interstitial pneumonia imaging pattern
}

\author{
To the Editor:
}

The introduction of antifibrotic agents to clinical practice and the need to better define patient populations for future clinical trials highlight the importance of accurate diagnosis. The main unmet need lies within the population of patients with fibrotic interstitial lung disease (f-ILD) and indeterminate for usual interstitial pneumonia (UIP) pattern on high-resolution computed tomography (HRCT). The differential diagnosis mainly includes idiopathic pulmonary fibrosis (IPF), nonspecific interstitial pneumonia (NSIP), ILD associated with occult collagen tissue disease and, most notably, chronic hypersensitivity pneumonitis.

The diagnostic role of bronchoalveolar lavage (BAL) in the population described is poorly defined and remains controversial even among experts [1-4]. According to the latest IPF diagnostic guidelines by the American Thoracic Society (ATS), European Respiratory Society (ERS), Japanese Respiratory Society (JRS) and the Latin American Thoracic Association (ALAT), for patients with newly detected ILD of apparently unknown cause who are clinically suspected of having IPF and have an HRCT pattern of probable UIP, indeterminate for UIP or an alternative diagnosis, cellular analysis of their BAL fluid (BALF) is suggested to distinguish IPF from some alternative ILDs, most notably eosinophilic pneumonia and sarcoidosis [5]. However, this recommendation is conditional and based on very low quality of evidence. Furthermore, the guidelines concluded that there is no difference in the proportion of BALF lymphocytes between patients with IPF and hypersensitivity pneumonitis. We aimed at exploring the added diagnostic value of BAL in patients with f-ILD and indeterminate-for-UIP pattern.

Patients with f-ILD and indeterminate HRCT pattern were identified retrospectively from a cohort of consecutive patients referred to the interstitial lung disease department of the First Respiratory University Clinic of the National and Kapodistrian University of Athens at the Chest Diseases Hospital Sotiria, between 2014 and 2017 (institutional review board: 587/7-12-18). These patients underwent BAL in our centre according to the latest ATS guidelines [6] and their initial diagnoses were re-evaluated by our multidisciplinary team. Two observers evaluated HRCT findings blinded to the BAL results and other clinical data and reached a consensus. Inclusion criteria were the presence of a fibrotic lung disease with an indeterminate-for-UIP pattern on HRCT according to the Fleischner criteria and the latest ATS/ERS/ JRS/ALAT guidelines for IPF [5,7]. Exclusion criteria were the absence of fibrotic changes on HRCT, the presence of a typical UIP or probable UIP pattern on HRCT, the presence of HRCT features most consistent with a non-IPF diagnosis according to the Fleischner definition [7], the presence of an already known diagnosis and when BAL was performed for investigation of infectious diseases.

Final diagnoses were established within the context of multidisciplinary discussion according to current guidelines [8]. Patients were considered to have unclassifiable ILD when review of clinical, radiological and pathological data did not reveal a specific diagnosis following multidisciplinary discussion [7-13]. Given the lack of diagnostic criteria regarding hypersensitivity pneumonitis, the latter was diagnosed based on the presence of an inciting antigen, compatible HRCT imaging, BALF lymphocytosis and new information during dynamic follow-up of the patients (identification of a previously unrecognised inciting antigen) [12-14].

Data were analysed statistically using MedCalc (version 14; MedCalc, Ostend, Belgium) and were expressed as mean \pm SD. Comparison of non-normally distributed variables between groups was done with one-way

@ERSpublications

BALF lymphocytosis is a valuable complementary tool in patients with fibrotic interstitial lung disease (f-ILD) and indeterminate-for-UIP pattern on HRCT http://bit.ly/2nCTkOh

Cite this article as: Tzilas V, Tzouvelekis A, Bouros E, et al. Diagnostic value of BAL lymphocytosis in patients with indeterminate for usual interstitial pneumonia imaging pattern. Eur Respir J 2019; 54: 1901144 [https://doi.org/10.1183/13993003.01144-2019]. 
ANOVA. Comparison of categorical variables between two groups was performed using the Chi-squared test. Differences were considered statistically significant at $\mathrm{p}<0.05$.

Among the 95 patients with undiagnosed f-ILD and an indeterminate-for-UIP pattern on HRCT, the majority were male $(70.5 \%)$ with mean \pm SD age $69.5 \pm 9.4$ years, and $69.5 \%$ were ex-smokers. Follow-up time was $980 \pm 481$ days. At baseline, forced vital capacity (FVC) was $75.3 \pm 18.7 \%$ predicted and $D_{\text {LCO }}$ was $53.8 \pm 20.2 \%$ pred. No significant differences were observed between patients with IPF, hypersensitivity pneumonitis and other forms of f-ILDs regarding age, sex, presence of comorbidities, pulmonary function test values (FVC, $D_{\mathrm{LCO}}$ ), need for supplementary oxygen and number of patients subjected to lung biopsy (table 1).

After re-evaluating all cases, final diagnoses included 35 patients with hypersensitivity pneumonitis, 19 patients with IPF and 41 patients with other forms of f-ILDs.

Almost one-third of patients $(n=32,33.7 \%)$ exhibited BALF lymphocytosis $\geqslant 20 \%$. Specifically, BAL lymphocytosis of $20-29 \%$ was observed in 15 (15.8\%) patients, $30-39 \%$ in seven $(7.4 \%)$ patients and $\geqslant 40 \%$ in $10(10.5 \%)$ patients.

The percentage of lymphocytes was significantly higher in the hypersensitivity pneumonitis group compared to the other groups $(\mathrm{p}<0.001)$ (table 1$)$. Notably, even when hypersensitivity pneumonitis diagnosis was based on non-BAL criteria (mostly exposure with suggestive computed tomography features, and biopsy in three cases), hypersensitivity pneumonitis was associated with a much higher prevalence of a BAL lymphocytosis $(\mathrm{p}<0.0005$, OR 4.9$)$.

Re-evaluation of patients driven by BAL results led to a change in diagnosis in $14(14.7 \%)$ patients. In the majority of these cases (11 (78.6\%) out of 14), the initial diagnosis was altered from IPF to hypersensitivity pneumonitis. In the remaining cases, the initial diagnosis was changed from unclassifiable fibrosis to hypersensitivity pneumonitis, from IPF to and from NSIP to hypersensitivity pneumonitis. Interestingly, identification of a previously unidentified inciting agent occurred in seven patients.

In the present study we evaluated the clinical utility of BAL in a specific population of patients with f-ILD depicting an indeterminate-for-UIP pattern on HRCT. Percentage of BAL lymphocytes was significantly higher in hypersensitivity pneumonitis compared to the other groups. The additional information provided from BAL results was clinically significant, as it led to a change of diagnosis in 14 (14.7\%) out of

TABLE 1 Baseline characteristics and bronchoalveolar lavage (BAL) cellular analysis of the study population ${ }^{\#}$

\begin{tabular}{lcccc} 
& Hypersensitivity pneumonitis & IPF & Other & p-value \\
\hline Subjects & 35 & 19 & 41 & \\
Age years & $69.7 \pm 9.3$ & $70.89 \pm 7.47$ & $68.76 \pm 810.29$ & 0.710 \\
Sex & & & & \\
$\quad$ Male & $25(71.4)$ & $16(84.2)$ & $26(63.4)$ & 0.796 \\
$\quad$ Female & $10(28.6)$ & $3(15.8)$ & $15(36.6)$ & 0.457 \\
Smoking status & & & & \\
$\quad$ Ex-smokers & $22(62.9)$ & $16(84.2)$ & $28(68.3)$ & 0.793 \\
$\quad$ Never-smokers & $13(37.1)$ & $3(15.8)$ & $13(31.7)$ & 0.461 \\
Comorbidities & & & & \\
$\quad$ GORD & $11(31.4)$ & $3(15.8)$ & $17(41.5)$ & 0.345 \\
$\quad$ Pulmonary hypertension & $7(20.0)$ & $3(15.8)$ & $7(17.1)$ & 0.934 \\
Arterial hypertension & $14(40.0)$ & $6(31.6)$ & $18(43.9)$ & 0.833 \\
Diabetes & $4(11.4)$ & $3(15.8)$ & $6(14.6)$ & 0.908 \\
Surgical lung biopsy & $8(22.9)$ & $5(26.3)$ & $5(12.2)$ & 0.463 \\
FVC \% pred & $78.06 \pm 18.85$ & $74.35 \pm 20.85$ & $72.81 \pm 17.54$ & 0.525 \\
DLco \% pred & $55.87 \pm 21.64$ & $48.06 \pm 17.62$ & $55 \pm 20.03$ & 0.411 \\
Supplementary oxygen & $5(14.3)$ & $3(15.8)$ & $6(14.6)$ & 0.992 \\
Macrophages \% & $61.66 \pm 15.84$ & $70.68 \pm 13.78$ & $68.05 \pm 14.64$ & 0.067 \\
Lymphocytes \% & $24.06 \pm 15.4 * *$ & $11.42 \pm 6.17 * * *$ & $13.9 \pm 11.32 * * *$ & $<0.001 * * *$ \\
Eosinophils \% & $3.69 \pm 3.23$ & $5.37 \pm 5.82$ & $5.01 \pm 5.14$ & 0.341 \\
Neutrophils \% & $10.24 \pm 11.02$ & $11.26 \pm 8.59$ & $11.66 \pm 9.84$ & 0.825 \\
\hline
\end{tabular}

Data are presented as $n$, mean \pm SD or $n(\%)$, unless otherwise stated. $p$-values were calculated using one-way ANOVA. IPF: idiopathic pulmonary fibrosis; GORD: gastro-oesophageal reflux disease; FVC: forced vital capacity; $D_{\text {LCO }}$ : diffusing capacity of the lung for carbon monoxide. \#: diagnoses are after re-evaluation incorporating BAL findings. ${ }^{* * *}: \mathrm{p}<0.001$. 
95 patients. Notably, in the majority of these cases the diagnosis was changed from IPF to hypersensitivity pneumonitis. Even if we exclude cases subjected to surgical lung biopsy, BAL changed the diagnosis in 10 cases, both by causing clinicians to identify an exposure $(n=7)$, so that non-BAL criteria were met, and by justifying a change in diagnosis in its own right, even when no exposure was found $(n=3)$. In this clinical setting, even a mild BAL lymphocytosis $(>20-25 \%)$ should increase vigilance for an underlying inciting antigen.

There are several limitations to our study. An obvious limitation is its retrospective nature. Another limitation is that it represents a single-centre study in a national reference ILD centre with the lack of an external cohort. We expect that the frequency of BAL lymphocytosis and subsequently its diagnostic value will vary significantly from centre to centre depending heavily on the local prevalence of hypersensitivity pneumonitis. In addition, all diagnoses were established by our local multidisciplinary team. It is known that inter-multidisciplinary team agreement is not ideal for non-IPF f-ILDs, especially hypersensitivity pneumonitis [15]. Finally, an inherent limitation of the study is incorporation bias, since the presence of BALF lymphocytosis pointed to diagnoses other than IPF. However, we tried to study the added value of BAL in clinical practice settings. BALF lymphocytosis was not considered as a gold standard for the diagnosis of hypersensitivity pneumonitis. In fact, in $40 \%$ of hypersensitivity pneumonitis patients, BALF lymphocytosis was $<20 \%$. Most importantly, when BAL was wholly excluded, hypersensitivity pneumonitis was still associated with a much higher prevalence of a BAL lymphocytosis.

In conclusion, BAL lymphocytosis is rather common in patients with no apparent diagnosis and indeterminate-for-UIP pattern on HRCT. Information provided by BAL (i.e. presence or absence of BAL lymphocytosis) is clinically useful in this clinical setting. BAL lacks the sensitivity and specificity to be used as a standalone diagnostic tool. However, our data strengthen the important complementary role of BAL in the diagnostic evaluation of f-ILDs and highlight the need for prospective, multicentric studies.

Vasilios Tzilas ${ }^{1,6}$, Argyrios Tzouvelekis ${ }^{1,6}$, Evangelos Bouros ${ }^{1}$, Theodoros Karampitsakos $\oplus^{2}$, Maria Ntasiou ${ }^{1}$, Mattheos Katsaras ${ }^{3}$, Ulrich Costabel ${ }^{4}$, Athol Wells ${ }^{5}$ and Demosthenes Bouros $\circledast^{1}$

${ }^{1}$ First Academic Dept of Pneumonology, Interstitial Lung Diseases Unit, Hospital for Diseases of the Chest, "Sotiria", Medical School, National and Kapodistrian University of Athens, Athens, Greece. ${ }^{2}$ Fifth Dept of Pneumonology, Hospital for Chest Diseases "Sotiria", Athens, Greece. ${ }^{3}$ Sixth Dept of Pneumonology, Hospital for Chest Diseases "Sotiria", Athens, Greece. ${ }^{4}$ Interstitial and Rare Lung Disease Unit, Ruhrlandklinik, University Hospital, Essen, Germany. ${ }^{5}$ Interstitial Lung Disease Unit, Royal Brompton Hospital, London, UK. ${ }^{6}$ Both authors contributed equally.

Correspondence: Demosthenes Bouros, Hospital for Chest Diseases "Sotiria", Messogion 152, Athens 11527, Greece. E-mail: debouros@gmail.com

Received: 11 March 2019 | Accepted after revision: 07 July 2019

Conflict of interest: V. Tzilas has nothing to disclose. A. Tzouvelekis has nothing to disclose. E. Bouros has nothing to disclose. T. Karampitsakos has nothing to disclose. M. Ntasiou has nothing to disclose. M. Katsaras has nothing to disclose. U. Costabel has nothing to disclose. A. Wells reports personal fees from Bayer AG, Roche and Boehringer Ingelheim, during the conduct of the study. D. Bouros reports grants, personal fees and non-financial support from Boehringer Ingelheim and Roche, and grants from Elpen, outside the submitted work.

\section{References}

1 Wells AU, Kokosi MA. Point: should BAL be routinely performed in the diagnostic evaluation of idiopathic pulmonary fibrosis? Yes. Chest 2017; 152: 917-919.

2 Mooney JJ, Collard HR. Counterpoint: should BAL be routinely performed in the diagnostic evaluation of idiopathic pulmonary fibrosis? No. Chest 2017; 152: 919-922.

3 Bouros D, Tzilas V, Tzouvelekis A. Diagnostic guidelines for IPF: when art meets science. Lancet Respir Med 2018; 6: 812-814.

4 Tzilas V, Valeyre D, Tzouvelekis A, et al. Taking a giant step in the diagnosis of idiopathic pulmonary fibrosis Lancet Respir Med 2018; 6: 82-84.

5 Raghu G, Remy-Jardin M, Myers JL, et al. Diagnosis of idiopathic pulmonary fibrosis. An official ATS/ERS/JRS/ ALAT clinical practice guideline. Am J Respir Crit Care Med 2018; 198: e44-e68.

6 Meyer KC, Raghu G, Baughman RP, et al. An official American Thoracic Society clinical practice guideline: the clinical utility of bronchoalveolar lavage cellular analysis in interstitial lung disease. Am J Respir Crit Care Med 2012; 185: 1004-1014.

7 Lynch DA, Sverzellati N, Travis WD, et al. Diagnostic criteria for idiopathic pulmonary fibrosis: a Fleischner Society white paper. Lancet Respir Med 2018; 6: 138-153.

8 Travis WD, Costabel U, Hansell DM, et al. An official American Thoracic Society/European Respiratory Society statement: update of the international multidisciplinary classification of the idiopathic interstitial pneumonias. Am J Respir Crit Care Med 2013; 188: 733-748.

9 Ryerson CJ, Urbania TH, Richeldi L, et al. Prevalence and prognosis of unclassifiable interstitial lung disease. Eur Respir J 2013; 42: 750-757.

10 Flaherty KR, King TE, Raghu G, et al. Idiopathic interstitial pneumonia: what is the effect of a multidisciplinary approach to diagnosis? Am J Respir Crit Care Med 2004; 170: 904-910. 
11 Travis WD, Hunninghake G, King TE, et al. Idiopathic nonspecific interstitial pneumonia: report of an American Thoracic Society project. Am J Respir Crit Care Med 2008; 177: 1338-1347.

12 Salisbury ML, Myers JL, Belloli EA, et al. Diagnosis and treatment of fibrotic hypersensitivity pneumonia. Where we stand and where we need to go. Am J Respir Crit Care Med 2017; 196: 690-699.

13 Vasakova M, Morell F, Walsh S, et al. Hypersensitivity pneumonitis: perspectives in diagnosis and management. Am J Respir Crit Care Med 2017; 196: 680-689.

14 Morisset J, Johannson KA, Jones KD, et al. Identification of diagnostic criteria for chronic hypersensitivity pneumonitis: an international modified Delphi survey. Am J Respir Crit Care Med 2018; 197: 1036-1044.

15 Walsh SLF, Wells AU, Desai SR, et al. Multicentre evaluation of multidisciplinary team meeting agreement on diagnosis in diffuse parenchymal lung disease: a case-cohort study. Lancet Respir Med 2016; 4: 557-565.

Copyright (C)ERS 2019 